\title{
La inclusión del público en la producción del contenido digital de los medios de comunicación
}

Public participation in the production of media digital content

\author{
Dick Ronald Cáceres Navarro* \\ Escuela Profesional de Ciencias de la Comunicación, \\ Universidad San Martín de Porres, Perú
}

\section{Resumen}

Facebook lidera las redes sociales con más usuarios a nivel mundial, en la actualidad tiene 2271 millones de cuentas activas. En el Perú cada mes se conectan 14 millones de personas a esta red social. Por este volumen de seguidores los medios de comunicación han encontrado en el uso de Facebook una forma exitosa de generar interacción con el público.

Para efectos de nuestra investigación tomamos como objeto de estudio la cuenta de Facebook del diario El Comercio, por la gran cantidad de seguidores (más de 3 millones) con los que cuenta y por la importancia histórica que tiene este medio de comunicación fundado hace 180 años. Mediante un sondeo de opinión, se explora la percepción de un grupo de lectores sobre la forma en que este diario, decano de la prensa nacional, gestiona la interactividad con sus seguidores en Facebook.

Palabras clave: redes sociales; medios de comunicación; interactividad; periodismo; Facebook 


\begin{abstract}
Facebook is the leading social network with the most users worldwide: it currently has 2.271 billion active accounts. In Peru, 14 million people log in to this platform every month. Considering this number of followers, the media has found in Facebook a successful way to interact with people. For the purposes of our research, the object of study will be the Facebook account of the El Comercio newspaper, due to its large number of followers (more than 3 million people) and the historical significance of this mean of communication founded 180 years ago. In the present research, we will analyze, through an opinion poll, what a group of readers think of the way in which Peru's longest established newspaper interacts with its followers on Facebook.
\end{abstract}

Keywords: social networks; media, interactivity; journalism; Facebook

\title{
Introducción
}

La presente investigación hace un repaso por el estado de la cuestión sobre la importancia de las redes sociales en el periodismo, la preponderancia que tiene la interactividad entre los medios de comunicación y sus usuarios, para demostrar que en la actualidad aún el aporte del público no está siendo completamente utilizado por los medios y que en el futuro se necesitará de una participación más activa del público en los formatos digitales de los medios informativos.

Según un estudio de la Universidad de Indiana el 40\% de los periodistas encuestados reveló que las redes sociales forman parte importante de su labor informativa, mientras que el $53.8 \%$ afirmó que recurre a estas para conseguir información.

Partimos de estos datos porque es innegable que la producción informativa ha cambiado radicalmente por las redes sociales, cuyo contenido es vital para el trabajo periodístico, sin embargo, creemos que los medios de comunicación masiva como el diario El Comercio de Perú, que tiene una cuenta de Facebook con más de 3.5 millones de seguidores, aún no aprovecha la interacción que sus lectores demuestran (con sus reacciones, compartidos y comentarios) para generar contenido de valor que incluya la visión del público en su versión digital. 


\section{Reseña del diario El Comercio}

El Comercio, conocido como el decano de la prensa escrita nacional, se fundó el 4 de mayo de 1839 por Manuel Amunátegui y Alejandro Villota, de nacionalidad chilena y argentina respectivamente.

Manuel Amunátegui tuvo que dejar la sociedad al ser deportado a finales de 1842, dejando toda la dirección del diario en manos de Alejandro Villota, quien en marzo de 1875 cedió la dirección a José Antonio Miró Quesada; su familia, hasta la fecha, tiene el control del grupo El Comercio.

Para acoplarse a los nuevos tiempos, en 1994 El Comercio sufre un importante proceso de rediseño el cual sentó las bases para la creación de los departamentos de infografía e ilustración. No es hasta 1997 que el diario incursiona en internet con la creación del dominio elcomercioperu.com.

Los redactores de la edición impresa de El Comercio recibieron de esta casa editora sus primeros smartphones en 2013 para empezar con la realización de contenido multimedia. El 1 de octubre de ese año se concreta la convergencia de plataformas, juntando en una misma redacción al personal de la edición impresa y digital. El 2 de noviembre de 2014, la redacción del diario hace uso del WhatsApp para obtener fotos, audio, videos y datos de sus usuarios.

\section{Facebook y Periodismo}

Facebook es la plataforma social más empleada por los cibernautas y también por los medios de comunicación, en el informe Digital In, elaborado por We are social digital (2019), se sostiene que Facebook lidera las redes sociales con más usuarios a nivel mundial. El estudio señala que tiene 2271 millones de cuentas activas

Para poner en contexto nuestra investigación y justificar el porqué escogimos la cuenta de Facebook de El Comercio como objeto de estudio, citaremos a la misma red social, que en su página oficial señala: En Perú la cantidad de usuarios mensuales sigue creciendo. Cada mes se conectan 14 millones de personas a la plataforma en ese país. Cada mes 14 millones de peruanos acceden a Facebook. 
Las redes sociales son el lugar idóneo para los hombres de prensa, ya que sus contenidos son visualizados por los usuarios generando su opinión y sus aportes informativos. «Muchos medios han notado que el volumen de comentarios del lector en una historia de Facebook puede exceder de los comentarios publicados en la web del periódico» (Cerviño, 2013, p. 35).

Nos quedamos con las últimas líneas expresadas por Cerviño para poner en relevancia que hoy en día los comentarios de Facebook en cualquier medio de información con un óptimo alcance pueden ser tomados por los medios para generar contenido en su formato digital.

El uso de Facebook por parte de los periodistas ha contribuido a generar una interacción con los usuarios, a diferencia de los foros que se usaban en los inicios del internet, la gran diferencia entre los foros de los primeros tiempos de internet y las redes sociales es que los primeros eran espacios concretos donde se planteaban debates y facilitaban intercambio de ideas. Sin embargo, las redes sociales, más que un espacio concreto en el que se reunen para hablar (como en los foros), son un espacio donde se dan una serie de interacciones entre individuos que comparten los mismos intereses, valores o creencias.

Las redes sociales han permitido la interrelación con usuarios que están en cualquier parte del mundo, acorta las barreras de la comunicación. Gutiérrez (2013) al respecto acota:

Para el periodista José Cervera, las redes sociales constituyen, la tertulia de Internet, sitios donde la gente intercambia información, donde hablan unos con otros. Hay dos vertientes interesantes que se producen dentro de las redes sociales, de un lado, la de mantener el contacto con otras personas, y de otro, la de aprender cosas nuevas. (p. 95)

Las plataformas sociales permiten a las empresas periodísticas llegar a los usuarios sin ninguna restricción geográfica, tomar su opinión y además sus aportes (textos, fotos, audios, videos, entre otros) y poder construir productos periodísticos con estos insumos informativos. 


\section{Hablemos de interactividad}

En teoría la interactividad hace que el usuario cuente con una participación activa en la creación de contenidos y exprese sus puntos de vista

Se puede definir a la interactividad como las propiedades que componen un medio para otorgar a los usuarios el dominio en la elección de contenidos y en las formas de expresarse o comunicarse, rompiendo los límites de los usuarios al hecho de ser solamente un observador de un programa multimedia. (Roggiero \& Vera, 2014, pp. 49$50)$

Hay que precisar además, que hoy gracias a la tecnología, los usuarios podrían tener una participación más activa que no se limite a emitir su opinión a través de una pantalla táctil o introducir texto en un comentario, el formato digital permite a cualquier medio de comunicación otorgar la facilidad a los usuarios (previamente registrados y filtrados) de tener un espacio en el cual puedan aportan contenido mediante alguna sección moderada por el editor periodístico.

Es cierto que actualmente, por medio de sus redes sociales, los medios propulsan el contacto y la retroalimentación, por ello Echevarría y Viada, (2014) señalan que «los medios online permiten a los usuarios participar a través de los comentarios, del envío de fotografías o videos sobre determinados hechos» (p. 18). Esto nos conduce a formular la siguiente pregunta: ¿será esto suficiente para una verdadera interactividad entre los medios de comunicación y sus usuarios en el futuro?

Una visión mucho más amplia, con respecto a la contribución de los usuarios en la generación de contenidos, nos la brinda Angulo (2016):

Es muy útil rescatar la distinción de formas de interactividad que hacen Díaz Noci y Palacios según el grado de intervención del usuario en la elaboración de contenidos dentro de los medios digitales. Desde esa perspectiva, la interactividad puede ser inclusiva (o "periodismo de código abierto», en el que los usuarios intervienen en los contenidos 
generalmente añadiendo cosas, como en los foros, o bien aportando información que sirve para organizar los contenidos que luego se le muestran) o autorial (la participación a los usuarios está permitida pero no hasta el punto de confeccionar contenido periodístico). (pp. 138-139)

En el Perú (y El Comercio no es la excepción) los espacios digitales de los grandes medios de comunicación hacen uso del periodismo de código abierto, publicando contenido enviado por los usuarios (principalmente fotos y videos), pero aún no le dan al público un espacio en sus webs para que estos se manifiesten, dejando de lado quizá una enriquecedora práctica de interactividad.

\section{Método}

Se realizó un sondeo de opinión a un grupo de seguidores del Facebook de el diario El Comercio, por ser el medio de comunicación de reconocido valor histórico en el país (Perú), y uno de los que más seguidores tiene en redes sociales.

El instrumento aplicado a los participantes de la investigación es un cuestionario, integrado por cuatro preguntas para recoger información mediante respuestas cerradas que se plantean de forma idéntica y homogénea, lo que permite su cuantificación y tratamiento estadístico.

Esta es una investigación aplicada porque se usa los conocimientos ya existentes como teorías, enfoques y principios en cada variable de estudio. Para el procesamiento de datos se utilizó el programa SPSS versión 21, con el cual se efectuó el análisis descriptivo de los ítems.

Para analizar la percepción que tiene el público de El Comercio sobre la interacción de este diario con sus seguidores de Facebook se realizó un sondeo de opinión entre 1000 usuarios de esta red social que siguen a El Comercio. Como se ha señalado se utilizó un cuestionario, mediante el cual se formuló las siguientes preguntas:

- ¿Cree que el diario El Comercio toma en cuenta sus sugerencias mediante Facebook? 
- ¿Considera que El Comercio utiliza correctamente su cuenta de Facebook para comunicarse con sus seguidores a través de mensaje directo, inbox o respondiendo a comentarios dejados por los usuarios?

- ¿Ha visto satisfecha sus necesidades informativas en la cuenta de Facebook de El Comercio?

- ¿Estaría de acuerdo con que en algún momento El Comercio brinde a sus lectores un espacio para expresar sus opiniones e información en su versión digital (web)?

El objetivo es conocer la percepción de los seguidores de este diario frente a sus comentarios, interacciones y sugerencias que realizan en Facebook.

\section{Resultados y discusión}

Frente a la pregunta: ¿Cree que el diario El Comercio toma en cuenta sus sugerencias mediante Facebook?, 94\% respondió que no y solo $6 \%$ respondió que sí. Al respecto, en un estudio realizado por Espinoza (2012), 80\% de los encuestados sostienen que las redes sociales influyen en la labor periodística, por tal razón la interacción con los usuarios es primordial en dichas plataformas.

Frente a la pregunta: ¿Considera que El Comercio utiliza correctamente su cuenta de Facebook para comunicarse con sus seguidores a través de mensaje directo, inbox o respondiendo a comentarios dejados por los usuarios?, 96\% respondió que no, mientras $4 \%$ respondió que sí. Estableciendo de esta manera la percepción de los participantes sobre lo que representa Facebook como canal efectivo de este diario para comunicarse con sus seguidores. Lo cual, es corroborado por Zubieta (2016), cuando asevera el interés del diario El Comercio por incursionar en las redes sociales Facebook y Twitter donde los redactores disputaban mayor participación. La tarea pendiente es el fomento de la bidireccionalidad en la comunicación con los usuarios.

Tenemos que destacar que, a pesar de que un gran porcentaje de los entrevistados piden una atención más fluida entre la cuenta de Facebook de El Comercio y sus seguidores, la gran mayoría de encuestados está conforme con la información periodística que recibe de este diario mediante su cuenta de Facebook. 
Respecto a la pregunta: $¿$ Ha visto satisfecha sus necesidades informativas en la cuenta de Facebook de El Comercio?, 77\% de los encuestados manifestó que sí y 3\% manifestó que no. Reafirmando lo mencionado por De Jesús (2015), quien señala que desde el 29 de abril de 2009, fecha en que El Comercio compartió su primer enlace vía Facebook, los usuarios mostraron interés por las diversas noticias publicadas, generando un gran tráfico en el espectro digital.

Finalmente, frente a la pregunta: ¿Estaría de acuerdo con que en algún momento El Comercio brinde a sus lectores un espacio para expresar sus opiniones e información en su versión digital (web)?,98\% de los participantes respondió afirmativamente.

Es decir, los lectores anhelan que el diario El Comercio brinde un espacio a sus lectores para que publiquen sus propios contenidos, con voz e imagen propia, como lo viene haciendo actualmente Canal $\mathrm{N}$, donde los usuarios elaboran sus propias informaciones prestando su imagen, pero mientras ello ocurra, el diario El Comercio ha dado el primer paso para la generación de contenido, así lo señala Zubieta (2016) cuando sostiene que los usuarios envían fotografías o videos de determinado hechos para su publicación en la página web.

\section{Conclusiones}

Se evidencia el interés de los usuarios, seguidores de Facebook del diario El Comercio, por tener una mayor actividad en la construcción informativa de tan importante medio de comunicación.

Parte del futuro de los medios de comunicación será brindar una participación más activa en sus formatos digitales al público para hacer un contenido más plural, democratizar el proceso informativo y fidelizar a las nuevas generaciones. 


\section{Referencias}

Angulo, H. (2016). Periodismo multimedia: El diseño multimedial en el periodismo digital boliviano. Punto Cero, (32), 51-75.

Cerviño, B. (2013). El uso de las redes sociales como fuente de información para periodistas (Tesis de maestría). Universidad Autónoma de Barcelona, España.

Echevarría, M., \& Viada, M. (2014). Periodismo en la web. Lenguajes y herramientas de la narrativa digital. Argentina: Editorial Brujas.

Espinoza, A. (2012). La cultura digital en el periodismo impreso: el caso del diario El Comercio (Tesis de licenciatura). Pontificia Universidad Católica del Perú, Lima.

De Jesús, N. (2015). Cómo cambian las redacciones de los medios periodísticos con la labor y desempeño laboral del Community Manager (Tesis de licenciatura). Universidad Antonio Ruíz de Montoya, Perú.

Gutiérrez, F. (2013). Los nuevos medios y el periodismo en medios sociales (Tesis de doctorado). Universidad Complutense de Madrid, España.

Roggiero, G., \& Vera, E. (2014). El periodismo 2.0 y su influencia en la migración de medios tradicionales en la web para la provincia de Santa Elena (Tesis de licenciatura). Universidad Estatal Península de Santa Elena, La Libertad, Ecuador.

We are social digital. (2019). Global Internet Use Accelerates 2019. Recuperado de https://wearesocial.com/ blog/2019/01

Zubieta, R. (2016). Gestión del contenido social y del reporterismo ciudadano en la producción de información para las secciones Lima y Perú del sitio web del diario El Comercio (Tesis de licenciatura). Universidad Nacional Mayor de San Marcos, Perú. 